\title{
Colloids, Nanostructures, and Supramolecular Assemblies for Papermaking
}

\begin{abstract}
Yongsheng Wang, Haiyan Huo, Xueren Qian, and Jing Shen *
The dominating role of colloid science in papermaking processes, as exemplified by wet-end chemistry, is now well known. The concept of colloids dates back to about 160 years ago. In certain cases, however, the term "colloids" can have an overlapping meaning with the modern terms "nanomaterials" and "supramolecular assemblies". The latter terms, and the scientists who have gravitated to those terms, have enriched colloid science, providing new insights into colloidal systems. It is proposed here that reconsidering papermaking in light of these multi-disciplinary sciences has potential to facilitate effective teaching and learning pertaining to universities that have pulp and paper programs. Enhanced integration of basic sciences with papermaking may further our understanding and broaden existing research areas, which is likely to create breakthroughs in basic research, applied research, and product development.
\end{abstract}

Keywords: Colloid science; Nanoscience; Supramolecular science; Wet-end chemistry; Teaching; Learning; Research; Papermaking

Contact information: Key Laboratory of Bio-based Material Science and Technology of Ministry of Education, Northeast Forestry University, Harbin 150040, China;

*Corresponding author: jingshen.china@ hotmail.com; jingshen@nefu.edu.cn

\section{The Need for a Deeper Look}

Papermakers contribute on a large scale to meeting human needs. Commercially, converting hydrodispersible fibers into paper-based products involves well-established, streamlined, and sustainable processes. Paper consumers are accustomed to diversified products for all end-uses. Research and development strategies are driving the industry toward new heights. Concurrently, however, Chinese universities with pulp and paper programs are facing challenges that impede further advancement. Exemplary obstacles include: (1) a limited percentage of Bachelor graduates engaged with such programs choose to work within this field (much less than 1/3, as in the case of Northeast Forestry University during the past five years); (2) domestic research activity in this field has been shrinking due to technological maturity, competitive funding, bars of promotion for high impact publications, etc. It may not be easy to change the current situation, since pulp and paper is a somehow narrow field. A deeper look at this field is necessary to unlock hidden features, enhance scientific interest, identify new possibilities of teaching and research, broaden the scope of applications, and further diversify the products. As an example, it is interesting to re-examine the basic sciences that govern papermaking processes.

\section{Colloids and Related Terms}

"Colloids", which was brought into scientific usage by Thomas Graham (a British scientist) (Graham 1861), is much older than the terms "nano" and "supramolecular". Having its origins in Greek ( $\kappa o ́ \lambda \lambda \alpha$, meaning glue), the word has long been associated with the science of turbid mixtures containing submicroscopic particles or droplets. The 
scientific field of colloids has been especially concerned with stability of the suspensions, short-range forces of interaction, and conditions leading to precipitation of the suspended matter. The term "nano" is most clearly identified with the size of the material. To qualify for placement in the "nanomaterial" category, at least one of the three dimensions of the material must be in a range between "larger than a typical molecule" and less than about $100 \mathrm{~nm}$. Notably, however, this is the range of distances over which most important colloidal interactions occur. The term "supramolecular" emphasizes processes of coming together of molecules by non-covalent interactions. The resulting structures are likely to be within the "nano-scale" of size, and the forces bringing about the assembly can be regarded as colloidal in nature. Thus, though emphasizing different aspects, the terms colloidal, nano, and supramolecular often can be used interchangeably.

\section{Colloids for Papermaking}

The dilute aqueous slurry running out of the headbox of a paper machine, with the possible exception of the fibers themselves, can be regarded as a good example of a colloidal system containing colloidal materials with varying characteristics. Notably, papermaking processes often start out with fine particles such as mineral fillers and emulsified sizing agents as stable, evenly dispersed particles or droplets. But at a critical point in the processes the papermaker needs the fine particles to adhere to fibers within the wet web of paper. To achieve this, the papermaker employs common tools of the trade of a colloid scientist - adding high-valence ions opposite in charge to the surfaces (e.g. aluminum sulfate or "alum"), as well as high-mass polyelectrolytes, to overcome the stabilizing forces and bring about deposition and adhesion among the solids. Even though papermakers have coined their own terms, such as "retention aid", to account for the resulting destabilization, it is clear that what they are really practicing is just a version of colloidal technology. It is worth noting that fibers are also conceivable as colloids or colloidal composites due to surface characteristics (e.g. colloidal surface fibrils).

\section{Papermaking's Nano Characteristics}

Despite the fact of largely involving the use of micrometer-sized fibers (i.e. fibers having diameters much larger than $100 \mathrm{~nm}$ ), papermaking also shows certain nano characteristics. The surface of papermaking fibers can include nanostructures due to the presence of fibrils. Mechanically and chemically liberated fiber fines can contain nanoscale particles. Nanoadditives, such as bentonite and colloidal silica, have found use in paper sheet formation. The interaction between oppositely charged polyelectrolytes can generate nano-sized complexes (Hubbe 2005; Arboleda et al. 2014). Functional nanostructures, once efficiently formed on the surface of fibers or anchored to fibers, are likely to result in the design of paper-based nanoproducts for tailorable applications.

\section{Supramolecular Assemblies for Papermaking}

Supramolecular science is a broad, multidisciplinary field concerning non-covalent bonding interactions of molecular building blocks and construction of molecular assemblies (Lehn et al. 1993; Vicens and Vicens 2011). This field is usually concerned with organized or well-defined structures of assemblies. The assemblies of greatest interest to supramolecular scientists are essentially colloids consisting of their own building blocks.

Papermaking, with its fully integrated industrial processes, involves the packing of micrometer-sized, hollow tube-like fibers into fiber-based supramolecular assemblies. 
Bonding interactions between neighboring fibers in such assemblies are largely based on hydrogen bonds. Fiber's surface molecules are responsible for supramolecular interactions, forming hydrogen-bonded fiber assemblies. During sheet consolidation (i.e. dryinginduced water vapor removal), hydrogen bonds between water and fibers are replaced by interfiber hydrogen bonds. These bonds are facilely reversible in the presence of water, which serves as the theoretical foundation for paper recycling.

Besides fiber-fiber interactions that contribute to the formation of hydrogen-bonded fiber assemblies, cellulosic fines and various types of additives mostly non-covalently interact with papermaking fibers in aqueous medium. These wet-end forces largely fit within the field of supramolecular science. Fine components and additives are anchored to, or aggregated with fibers, on the basis of forces such as electrostatic "binding", bridginginduced flocculation, and hydrogen bonding, generating "composite" fibers. Molecular reassembly/reorientation would come into play in process units following sheet formation, as in such cases as internal sizing (i.e. hydrophobic moieties of sizing molecules point toward the outside of fiber assemblies).

The dilute suspension containing fibers and other components for papermaking is an economically important example of industrial colloidal systems. Strategies for tailoring the behavior of colloids involve much knowledge of supramolecular science. Emulsions such as those based on rosin and alkylketene dimer are stabilized in light of molecular assembly at the interfaces. Formation of supramolecular polyelectrolyte complexes between cationic polymers (e.g. cationic starch) and anionic polymers (e.g. dissolved hemicelluloses) in papermaking systems is a possible phenomenon. Such complexes can be designed to improve wet end performance (Hubbe 2005; Arboleda et al. 2014). Hostguest based supramolecular interactions, induced by guest molecules (fatty acid salts or rosin acid salts), can facilitate the anchorage of starch to mineral particles to enhance filler bondability with cellulosic fibers (Yoon and Deng 2006; Huang et al. 2015).

Indeed, supramolecular phenomena are involved throughout the key processes of paper manufacturing practices, and the design of wet end chemical programs with enhanced performance can benefit from the integration of supramolecular science into process engineering. Papermaking science is definable as the supramolecular science associated with the use of fibers and other components to construct fiber-based complex assemblies, and reversible bonding interactions play a governing role. Paper-based products may be considered as fiber-based supramolecular products. Another interesting point is that well-established papermaking-based supramolecular assembling strategies are designable to produce paper-like materials with advanced functionalities, from liquiddispersible particles (Dikin et al. 2007).

\section{Future Opportunities}

In teaching practices, highlighting interesting scientific features of papermaking in light of colloid science, nanoscience, and supramolecular science would help to attract the interest of university students to become more engaged in pulp and paper programs. A student having a background in nanotechnology or supramolecular science may discover that papermaking technology is an ideal area in which to try out their ideas on a grand scale. The three subfields of multi-disciplinary sciences - colloids, nanotechnology, and the study of supramolecular assemblies - can serve as the foundation for gaining a deeper understanding of unit operations of papermaking, facilitating the identification of new possibilities of basic research, applied research, and product development. 


\section{Acknowledgements}

This work was supported by the Fundamental Research Funds for Central Universities of China (2572018CG04), the Natural Science Foundation of China (218708046), the Program for New Century Excellent Talents in University (NCET-120811), and the Longjiang Scholars Program (Q201809). We greatly appreciate the constructive editing help from Prof. Martin A. Hubbe (North Carolina State University) for insightful comments and suggested changes.

\section{References Cited}

Arboleda, J. C., Niemi, N., Kumpunen, J., Lucia, L. A., and Rojas, O. J. (2014). “Soy protein-based polyelectrolyte complexes as biobased wood fiber dry strength agents," ACS Sustainable Chem. Eng. 2(10), 2267-2274. DOI: 10.1021/sc500399d

Dikin, D. A., Stankovich, S., Zimney, E. J., Piner, R. D., Dommett, G. H. B., Evmenenko, G., Nguyen, S. T., and Ruoff, R. S. (2007). "Preparation and characterization of graphene oxide paper," Nature 448(7152), 457-460. DOI: 10.1038/nature06016

Graham, T. (1861). "Liquid diffusion applied to analysis," Proc. R. Soc. London 11, 243247. DOI:10.1098/rspl.1860.0048

Huang, X., Qian, X., Li, J., Lou, S., and Shen, J. (2015). "Starch/rosin complexes for improving the interaction of mineral filler particles with cellulosic fibers," Carbohydr. Polym. 117, 78-82. DOI: 10.1016/j.carbpol.2014.09.047

Hubbe, M. A. (2005). "Dry-strength development by polyelectrolyte complex deposition onto non-bonding glass fibres," J. Pulp Pap. Sci. 31(4), 159-166.

Lehn, J. M. (1993). "Supramolecular chemistry," Science 260(5115), 1762-1763. DOI: $10.1126 /$ science. 8511582

Vicens, J., and Vicens, Q. (2011). "Emergences of supramolecular chemistry: From supramolecular chemistry to supramolecular science," J. Inclusion Phenom. Macrocyclic Chem. 71(3-4), 251-274. DOI:10.1007/s10847-011-0001-z

Yoon, S. Y., and Deng, Y. (2006). "Starch-fatty complex modified filler for papermaking," Tappi J. 5(9), 3-9. 